\title{
Research progress on radiotherapy technology and dose fraction scheme for advanced gliomas
}

\author{
Yu Zhang ${ }^{1}$, Junjie Wang ${ }^{2} \wedge$ \\ ${ }^{1}$ Department of Radiation Oncology, Peking University International Hospital, Beijing, China; ${ }^{2}$ Department of Radiation Oncology, Peking \\ University Third Hospital, Beijing, China \\ Contributions: (I) Conception and design: All authors; (II) Administrative support: Yu Zhang; (III) Provision of study materials or patients: All authors; \\ (IV) Collection and assembly of data: All authors; (V) Data analysis and interpretation: J Wang; (VI) Manuscript writing: All authors: All authors; (VII) \\ Final approval of manuscript: All authors. \\ Correspondence to: Junjie Wang, PhD. Department of Radiation Oncology, Peking University Third Hospital, No. 49 of Huayuan North Road, \\ Haidian District, Beijing 100191, China. Email: wangjunjie_jundr3@163.com.
}

\begin{abstract}
Glioma is the most common central malignant tumor. High-grade glioma (HGG) has high malignancy and a short median survival. Complete surgical resection and comprehensive treatment with postoperative radiotherapy and chemotherapy is the recommended treatment for HGGs at present in clinic. Postoperative radiotherapy can reduce the local recurrence rate and prolong the survival time of patients. In recent years, researchers have made some progress on different radiotherapy technologies and dose fraction schemes. With the continuous development of medical technology, different groups of people should choose different dose fraction schemes, in order to realize the individualization of treatment schemes, and provide more benefits to patients. At present, the optimal radiotherapy dose, the fraction model, and how to achieve individualized radiotherapy remains unclear. In view of the poor prognosis of this disease, patients should be encouraged to participate in properly conducted experimental studies.
\end{abstract}

Keywords: Glioma; radiotherapy; chemotherapy; malignant tumor; dose splitting strategy

Submitted Apr 21, 2020. Accepted for publication Aug 28, 2020.

doi: $10.21037 /$ tcr-20-1891

View this article at: http://dx.doi.org/10.21037/tcr-20-1891

\section{Introduction}

Glioma is the most common intracranial tumor, which accounts for approximately $50 \%$ of primary intracranial tumors in adults (1). High-grade gliomas (HGGs, WHO grade III-IV) include anaplastic astrocytoma, anaplastic oligodendrocytoma and glioblastoma (GBM). The annual incidence of HGGs in the world is 6/100,000 (2), these tumors progress, and overall outcomes have not changed much in the past decade. The two most common subtypes of HGGs are anaplastic astrocytoma and GBM, which account for more than $80 \%$ of HGGs (3), and the 5 -year survival rate is $27 \%$ and $5 \%$ (4), respectively. Complete surgical resection and comprehensive treatment with postoperative radiotherapy and chemotherapy is the recommended treatment for HGGs at present. The European Organisation for Research and Treatment of Cancer-National Cancer Institute of Canada (EORTCNCIC) randomized phase III trial published in 2005 confirmed the role of the concomitant and adjuvant addition of temozolomide (TMZ) to radiotherapy $(5,6)$. Radiation therapy occupies an important role in treating gliomas, with the development of new technologies, combination of radiotherapy and multiple imaging modalities will increase the diagnostic accuracy and treatment efficiency of the area at a high-risk of relapse. However, the current situation of

^ ORCID: 0000-0002-3569-8459. 
HGGs therapy faced a choice between efficacy and toxicity. In recent years, researchers have made some progress on different radiotherapy technologies and dose fraction schemes. The present review summarizes the research progress of radiotherapy technology and the dose fraction scheme for HGGs. The details are reported, as follows.

\section{The development and application of radiotherapy technology}

Radiotherapy is one of the important treatment methods for HGGs. Radiotherapy performed within 6 weeks after the operation can significantly reduce the local recurrence rate, and prolong the survival of patients (7). With the continuous development of modern radiotherapy technology, radiotherapy regimens for HGG patients have become increasingly accurate, and the clinical effect has also been significantly improved.

\section{Importance of intensity-modulated radiation therapy (IMRT)}

With the development of radiotherapy technology, a therapy plan can significantly reduce the radiation dose for normal brain tissues $(8,9)$. At present, IMRT and volumetric modulated arc therapy (VMAT) are the most commonly used radiotherapy technologies for HGGs. IMRT can adjust the output of a standard linear accelerator (LINAC) according to the software, in order to change the radiation intensity across each treatment field. Compared with threedimensional conformal radiation therapy (3D-CRT), IMRT can increase the target coverage and conformability, reduce the dose of organs at risk, and accordingly reduce adverse reactions (10). Previous studies have revealed that (11-13) patients with HGGs undergoing IMRT presented with low doses in the brain, brainstem and optic chiasm, and a high target coverage, while IMRT did not increase the survival of these patients.

VMAT is one of the IMRTs, in which the radiation source arc rotates around the patient, and emits rays for the radiotherapy. Previous studies have revealed that $(14,15)$ compared with IMRT, VMAT is better in terms of delivery, and the coverage of the target areas of these two are equal. Furthermore, it reduces the machine monitor unit and radiotherapy time, improves the effect and comfort of the treatment. However, there is presently no consensus on which is more advantageous in protecting organs at risk between VMAT and IMRT.

\section{Proton radiotherapy}

Proton therapy is a commonly used form of particle therapy. Protons have unique physical properties. The application results of proton therapy for different tumors have been confirmed in phase I and phase II clinical trials (16-18). Compared with photon radiotherapy, the proton has a Bragg peak, a better dose distribution in tumors, a higher biological effect, and a lower dose to normal tissues. Local recurrence is the main failure model of HGGs after treatment, and local treatment, which mainly include a secondary operation and re-radiotherapy, is the main treatment for recurrent patients. However, the treatment effect is poor. Furthermore, it induces many complications after treatment, which affects the quality of life of patients, thereby preventing most patients with postoperative recurrence from obtaining an effective treatment. Verma et al. (19) investigated the safety and effectiveness of proton radiotherapy for recurrent patients, and it was suggested that proton radiotherapy can be used as an effective rescue treatment for recurrent patients. However, the late toxicity remains to be investigated. Adeberg et al. (20) conducted a study on 66 HGG patients, who were treated with conventional postoperative radiotherapy (50 Gy, 2 Gy/f), and subsequently treated with an added proton dose (an equivalent biological dose of $10 \mathrm{~Gy}, 2 \mathrm{~Gy} / \mathrm{f}$ ). Compared with the conventional treatment group (60 Gy, 2 Gy/f), the progression-free survival (PFS) and overall survival (OS) were similar. Furthermore, in the proton dosage group, the planning target volume (PTV) was significantly decreased $(\mathrm{P}<0.001)$, and the third-grade side effect was milder. A study on six centers was conducted by Vora et al. (21), which had the largest population on HGG proton radiotherapy. In this study, 63 patients were treated from 2009 to 2017, and these patients were followed up (73\% of patients were at $\mathrm{WHO}$ grade IV, and $27 \%$ of patients were at $\mathrm{WHO}$ grade III), with a median follow-up duration of 15 months. Among these patients, $89 \%$ of these patients received TMZ chemotherapy during the proton therapy, and the mean dose of the proton radiotherapy was $59.4 \mathrm{GyE}$ (40-66 GyE/ 15-33 f). Furthermore, the median OS was 18.3 months, the 2 -year OS was $39 \%$, and $>3$ levels of toxicity reaction occurred in three patients. These results reveal that proton 
radiotherapy has good tolerance, and its prognosis is similar to that of traditional therapy.

\section{The development of imaging technology and HGG radiotherapy}

Malignant glioma has no capsule, and infiltrative growth is one of its characteristics. The growth characteristics of HGGs make it difficult to determine the boundary between the tumor and the normal tissue. Pathology has also confirmed that scattered tumor cells infiltrating along the new blood vessel can be found in the peripheral edema area (22). Magnetic resonance imaging (MRI) is the most commonly used examination method for HGGs. In recent years, MRI technologies, including diffusion-weighted imaging (DWI), diffusion tensor imaging (DTI) and magnetic resonance spectroscopy (MRS), have been widely used in HGG radiotherapy. The development of other new examination technologies, such as positron emission tomography (PET), has also brought new progress to the radiotherapy of HGGs.

\section{$D W I$}

DWI reveals lesions by detecting the diffusion of water molecules and the apparent diffusion coefficient (ADC). A literature (23) considered that the lower the ADC value is, the higher the cell density and proliferation activity are. Therefore, areas with a low ADC value have high tumor density and obvious infiltration. Another study (24) revealed that DWI can help to define the boundary of glioma and its surrounding infiltration, and the accuracy of the HGG target delineation can be improved by increasing the dose of the high-risk area, according to the changes in the DWI image and ADC value. Park et al. (25) delineated the target areas of HGG patients according to the ADC and MRI-T1. They reported that the dose to the optic nerve and brainstem in the ADC-IMRT plan was reduced by $10 \%$ and $16 \%$, respectively.

DTI is one of the DWIs, which can evaluate the structure and physiological state of tissues by detecting the diffusion of water molecules. Anisotropic fraction (FA) is the most commonly used index of DTI. The decrease in FA value reflects the abnormality of white matter tracts. DTI can be used to distinguish between low-grade gliomas and HGGs, and it can also be used to distinguish between gliomas and brain metastasis (26). Jena et al. conducted a study (27), in which conventional radiotherapy and a radiotherapy plan with reference to the DTI were compared in HGG patients. The mean PTV of the target area in the DTI radiotherapy plan decreased by $35 \%(18-46 \%)$, and the toxicity in the two groups was similar during the treatment. Therefore, they considered that the application of DTI technology is expected to achieve individualized radiotherapy, and that the curative effect may be more significant. However, further studies are needed to determine how to make it play a better role. In a study conducted by Bian et al. (28), the DTI and FA measurement of the bilateral hippocampus were performed on 23 HGG patients before and after the radiotherapy, and the results revealed that after radiotherapy, the FA values of the bilateral hippocampus decreased, and this decrease in FA was prior to the occurrence of cognitive impairment. Therefore, DTI and FA value measurement of the hippocampus may be an effective examination method for radiation-induced neurocognitive impairment.

\section{MRS}

MRS is an MRI technology that can reflect the disease situation by detecting the concentration of metabolites in the body. The substances detected by MRS mainly include creatine (Cr), choline (CHO), lipid (LIP), lactic acid (LAC), acetyl aspartic acid (NAA), etc. The CHO signal reflects the formation and change of the cell membrane. NAA is a neural marker, which reflects the integrity of neurons (29). A previous study (30) revealed that compared with normal brain tissues, for HGGs, the CHO value was elevated, but the NAA and Cr values decreased. Furthermore, the sensitivity of MRS to tumor invasion was better, when compared to traditional enhanced MRI. MRS can also be used to distinguish between brain injury and tumor recurrence caused by radiotherapy. A study reported that (31) the sensitivity and specificity of CHO/NAA and $\mathrm{NAA} / \mathrm{Cr}$ for tumor recurrence were $86 \%$ and $90 \%$, and $93 \%$ and $70 \%$, respectively. However, further studies are needed to determine how MRS guides radiotherapy and target delineation.

\section{PET/computed tomography (CT)}

PET can remedy the shortcomings of MRI and other anatomical images by reflecting the HGG situation from molecular and metabolic aspects. The most commonly used contrast in PET is ${ }^{18} \mathrm{~F}$-fluorodeoxyglucose $\left({ }^{18} \mathrm{~F}\right.$-FDG). However, due to the high intake of glucose by the brain, 
${ }^{18} \mathrm{~F}-\mathrm{FDG}$ is not sensitive enough to produce the imaging picture of glioma. Other contrasts include amino acids, choline, acetic acid, nucleic acid, neuroreceptors, and amino acids is more commonly used than others. Compared with ${ }^{18} \mathrm{~F}$-FDG, amino acids, including ${ }^{11} \mathrm{C}$-methionine $\left({ }^{11} \mathrm{C}-\mathrm{MET}\right), \mathrm{O}-\left(2-\left[{ }^{18} \mathrm{~F}\right]\right.$ fluoroethyl $)$-L-tyrosine $\left({ }^{18} \mathrm{~F}-\mathrm{FET}\right)$, can show the proliferation and metabolism of the tumors at the metabolic and receptor level, and it's absorbed more in the tumor than normal tissues, thus is better in the imaging of glioma (32). ${ }^{18}$ F-FET has longer half-life (109.8 min) than ${ }^{11} \mathrm{C}-\mathrm{MET}$, so it's more used in clinic (33-35). Munck et al. (36) performed ${ }^{18} \mathrm{~F}$-FET PET and MRI scans on 54 HGG patients, and delineated the target areas. The volume of the target area based on the PET was larger in WHO grade IV patients $(\mathrm{P}<0.001)$, but smaller in patients with larger and more complete surgical resections $(\mathrm{P}=0.004)$. Therefore, researchers have speculated that target areas based on the PET may benefit patients at grade IV. However, this needs to be verified through a randomized prospective study.

Target volume definition for HGG during initial radiotherapy may yield significantly differing results depending upon the imaging modality. CT and MRI can show bony structure and soft tissue, respectively, and the combination of PET with CT or MRI can better distinguish tumor and normal brain tissue. PET/MRI fusion is more comprehensive than single examination in the diagnosis of glioma (37) and can more accurately define the target area, thereby making the radiotherapy of HGG patients more accurate. Researchers compared the radiotherapy plans of 44 patients with recurrent HGG, and found that the survival time of patients treated with the PET/MRI fusion radiotherapy plan was longer, when compared to patients treated with the CT/MRI radiotherapy plan (9 vs. 5 months) (38).

\section{Dose fraction scheme}

Fractionated external irradiation is the standard treatment for HGGs. Its survival rate is almost twice as surgery alone (39), but whether and how radiation therapy should be applied depends on characteristics specific to tumor and patient, including age and performance status. The suggested dose for HGGs is $60 \mathrm{~Gy} / 30 \mathrm{f}$, or $59.4 \mathrm{~Gy} / 33 \mathrm{f}$; for tumor with larger size or WHO grade III astrocytoma, dose can be decreased to 55.8-59.4 Gy, $1.8 \mathrm{~Gy} / \mathrm{f}$, or $57 \mathrm{~Gy} / 30 \mathrm{f}$. If boost dose is used, radiation dose can be increased by 14 Gy (2.0 Gy/f) or 9-14.4 Gy (1.8 Gy/f) after treatment at 46 Gy (2 Gy/f) or 45-50.4 Gy (1.8 Gy/f) (40). When depicting gross tumor volume (GTV), it's suggested to use MRI T1 enhanced phase and T2 fluid-attenuated inversion recovery (FLAIR) phase. For WHO grade III tumors, clinical target volume (CTV) is 1-2 cm outside GTV, but for GBM, CTV is $2-3 \mathrm{~cm}$ outside GTV. However, HGGs have high malignancy and a high postoperative recurrence rate, and $90 \%$ occurs within $2 \mathrm{~cm}$ of the primary tumor, so it's important to optimize the local radiotherapy (41). Researchers found that when split dose remains the same, increasing total dose does not bring more benefit to patients (42). Therefore, researchers have explored various fraction methods and radiotherapy regimens, including stereotactic radiosurgery (SRS), stereotactic radiotherapy (SRT), hypofractionated radiotherapy (HFRT), simultaneous integrated boost IMRT (SIB-IMRT).

\section{SRS}

The radiation sensitivity of $\mathrm{HGG}$ remains unsatisfactory. In theory, it is better to use a large dose. SRS is a single high-dose radiotherapy technology, which is mostly used for small intracranial lesions. In 2004, RTOG9305 published the results of a multicenter randomized controlled trial (43). In this trial, patients with GBM were randomly divided into two groups: one group of patients received conventional radiotherapy (60 Gy/30 f), while the other group of patients received routine external radiation immediately after treatment with SRS (15-24 Gy/f). Patients in both groups were treated with carmustine chemotherapy. The results revealed that there was no significant difference in median survival time. However, this trial was discussed, because SRS was conducted before external radiation, and carmustine was not used as a routine treatment in GBM patients. Some subsequent retrospective studies on SRS revealed that SRS can be used as a safe and effective treatment for HGG patients with small recurrent tumors (44-46). Morris et al. (47) reported that 45 patients with recurrent GBM were treated with SRS and bevacizumab. The median tumor volume was $2.2 \mathrm{~cm}^{3}$, and the average dose of SRS was 17 Gy (13-24 Gy), PFS and OS were 5.2 and 13.3 months after SRS, respectively. Abbassy et al. also reported that (48) recurrent GBM patients treated with SRS and bevacizumab, PFS and OS were 7.5 and 13 months, respectively. These studies reveal that SRS is beneficial for increasing the dose of HGG radiotherapy, and provides a new treatment approach for recurrent HGGs. However, in these studies, 
the sample sizes were small. The best population to benefit from SRS, the choice of the best dose of treatment, and the determination of when to intervene the SRS needs to be determined through further studies.

\section{$S R T$}

SRT is a non-coplanar, multi-field, $3 \mathrm{D}$ and multifractionated radiation mode developed on the basis of SRS. SRT is similar to SRS in terms of dose, but the dose gradient is not as large as that of SRS. Therefore, compared to SRS, SRT can be used to treat large tumors and multiple fractionated radiation can also reduce the radiation damage to normal tissues (49). Compared with the conventional treatment regimen, SRT can reduce the treatment time to 2 weeks and the shortened treatment time would significantly improve the quality of life of patients.

The RTOG0023 (50) included 76 patients with GBM, and these patients were treated with conventional fractionated radiotherapy with a total dose of 50 Gy. SRT (5-7 Gy/f) was added weekly during the 3-6 weeks of treatment. The median survival was 12.5 months and had no significant survival benefit when compared with the historical RTOG data. Many studies after RTOG0023 suggest that for relapse and elderly HGG patients, the benefits of SRT would be more significant. Fogh et al. investigated (51) the toxicity and efficacy of SRT (35 Gy/10 f, $3.5 \mathrm{~Gy} / \mathrm{f}$ ) in 147 patients with recurrent HGGs. The results revealed that SRT was well-tolerated and the median survival time after SRT was 11 months. Young patients, patients with small GTV sizes, and patients with short intervals between diagnosis and recurrence may have significant survival benefits. SRT is recommended for HGG patients who relapse within 6 months after traditional treatment. RTOG1205 (52) is the first multicenter, randomized controlled phase II clinical trial for recurrent GBM. Patients in this trial were divided into two groups: SRT (35 Gy/10 f, 3.5 Gy/f) + bevacizumab (10 mg/kg, once every 2 weeks) group and bevacizumab alone $(10 \mathrm{mg} / \mathrm{kg}$, once every 2 weeks) group. A total of 182 patients were enrolled in this study. There was no significant difference in median survival time between these two groups. In the SRT + bevacizumab group, PFS increased to 6 months (54\% vs. 29\%; HR 0.42, 95\% CI: 0.34-0.50, P=0.001).

\section{HFRT}

HFRT pertains to the administration of a large fractionated dose in a short time, and a single fractionated dose is greater than $2.5 \mathrm{~Gy} / \mathrm{f}$ (for example, $60 \mathrm{~Gy}$ is fractionated into 15-20 times). HFRT is more frequent, and has fewer single doses, when compared to SRT. Various studies have revealed that elderly patients with GBM can benefit from HFRT. In the Nordic Trail (53), 342 elderly patients were randomly assigned to three groups: TMZ treatment (200 mg/m², d1-d5, q28d) group, HFRT (34 Gy/10 f) group, and conventional radiotherapy $(60 \mathrm{~Gy} / 30 \mathrm{f})$ group. The OS of patients in the TMZ group and HFRT group was similar (8.4 vs. 7.4 months), and this significantly improved, when compared to that in the conventional radiotherapy group (6 months). This study suggests that conventional radiotherapy is not the best choice for elderly patients, especially for patients $>70$ years old. Furthermore, TMZ and HFRT should be used as a standard treatment for elderly GBM patients. Biau et al. (54) conducted a study on elderly patients with GBM, who were treated with HFRT + TMZ. This study also confirmed that HFRT + TMZ can benefit elderly patients with GBM, and TMZ treatment was recommended, regardless of the methylation of the $\mathrm{O}^{6}$-methylguanine-DNA-methyltransferase (MGMT). Roa et al. (55) conducted a study on 98 GBM patients (aged or frail), who were randomly divided into two groups (25 Gy/5 f and $40 \mathrm{~Gy} / 15 \mathrm{f}$ ). The differences in OS, PFS and quality of life between these two groups were not statistically significant. The median survival time was 7.9 and 6.4 months, respectively. Considering the treatment time, the $25 \mathrm{~Gy} / 5 \mathrm{f}$ regimen was preferred. In the EORTC/NCIC/Trans-Tasman Radiation Oncology Group (TROG) phase III clinical trial (56), 562 GBM patients (over 65 years old) were randomly divided into two groups: HFRT (40 Gy/15 f) group and HFRT + TMZ group. The OS of patients in these two groups was 7.6 and 9.3 months, respectively, and the PFS was 3.9 and 5.3 months, respectively. The differences were statistically significant. This study concluded that HFRT combined with TMZ can be recommended as a standard treatment for new GBM in the elderly.

\section{$S I B$}

Glioma has the characteristics of recurrence in situ, and its local recurrence is closely correlated to the survival of HGG patients (10). SIB-IMRT can irradiate different doses to different targets at the same time without increasing the toxicity to normal tissues (57). Cho et al. (58) treated 40 HGG patients with SIB-IMRT. The PTV dose per time 
was 2.0 Gy, the planning gross tumor volume (PGTV) dose per time was $2.4 \mathrm{~Gy}$, a total of 25 times of treatment was given. The median survival time was 14.8 months. It has been proven that SIB-IMRT is safe and feasible, and that its survival is equivalent to that of traditional radiotherapy. However, the best fractionated dose and its effect still needs to be further explored. Some scholars (59) have studied the maximum tolerable dose of SIB in HGG patients. The dose for PTV1 (operated cavity and residual tumor) started from $2.8 \mathrm{~Gy}$, and was increased in each group by $0.4 \mathrm{~Gy}$, in order to carry out a dose climbing test. A total of $20 \mathrm{f}$ was set. PTV2 (PTV1 $+2 \mathrm{~cm})$ was given at $2.5 \mathrm{~Gy}$ at a time, for a total dose of $50 \mathrm{~Gy}$, TMZ was concurrently given during the treatment. The results reveal that the maximum single-tolerance dose of SIB when combined with TMZ was 3.6 Gy/f (72 Gy/20 f), and that the median OS and PFS were 19 and 16 months, respectively. In the phase II clinical study conducted by Mallick et al. (60), 89 GBM patients, who were treated from 2011 to 2017, were randomly divided into two groups: traditional radiotherapy group (60 Gy/30 f) and SIB group. In the SIB group, a single dose per time was 3 Gy for highrisk PTV and 2.5 Gy for low-risk PTV, and a total of 20 fractions were given. The median OS of patients in the two groups was similar (18.07 vs. 25.18 months, $\mathrm{P}=0.3$ ), patients $<40$ years old with complete resection and isocitrate dehydrogenase 1 (IDH1) mutation had better OS. After 2 years, $60 \%$ of patients in the SIB group survived, while merely $24 \%$ of patients in the traditional radiotherapy group survived. After 2 years, $30 \%$ of patients in the SIB group did not progress, while patients in traditional treatment group all progressed. In an ongoing phase III clinical trial (61), GBM patients were divided into two groups: conventional STUPP treatment group and SIB radiotherapy combined with TMZ group. In the SIB group, the target area was delineated according to MRS. When $\mathrm{CHO} / \mathrm{NAA}$ was $>2$, the dose for the operated cavity and MR-enhanced area were simultaneously added. The single dose was $2.4 \mathrm{~Gy}$, and the total dose was $72 \mathrm{~Gy}$ (30 fractions). These results were as expected.

\section{Biomarkers}

Prognosis of HGG is related to many factors, including age, surgery, Karnofsky Performance Scale (KPS) score and pathology results. Detection of biomarkers can provide more information $(62,63)$. MGMT is very important in the decision of treatment regimen. MGMT is a DNA modification enzyme; methylation of its promoter can decrease its expression; thus, DNA reparation will be prevented, and the tumor will become sensitive to chemotherapy. Hegi et al. found that methylation of its promoter in patients can lead to better treatment results and prognosis of TMZ (64). Patients with IDH1/2 mutation usually also have MGMT promoter methylation (65-67). ATRX ( $\alpha$ thalassemia/mental retardation syndrome $\mathrm{X}$-linked) mutation is the biomarker of neuroastrocytoma; patients with this mutation have better prognosis. Other biomarkers include telomerase reverse transcriptase (TERT) mutation, epithelial growth factor receptor (EGFR) mutation, P53 mutation, and all have diagnosis values.

\section{Radiation toxicity}

Radiation toxicity include early and late stage toxicity, the former being reversible, which the latter being irreversible. Radiation will kill the newborn neuros, causing the loss of brain functions. Early toxicity of fractionated external irradiation include fatigue, anorexia, dermatitis, cephalgia, nausea. Late effects include radiation necrosis, neuroendocrine dysfunction, cognition disorders (68). Precise radiation has greatly decreased the occurrence of radiation toxicity after HGGs. The factors that can affect radiation toxicity include the volume of normal tissues that are involved in the radiation, total dose and fraction methods, tolerance of the patients, etc. HGG patients have a short median survival and high rate of recurrence at the original site. Many of the therapeutic strategies tend to increase the radiation dose to the target therefore increased the risk of radiation damage to the nearby normal brain structures, which would be associated with toxicity or even shortened survival (69-73).

\section{Summary}

HGGs are common central malignant tumors in clinic, which have the characteristics of high malignancy, short median survival time and high recurrence rate. Surgery, radiation, and chemotherapy remain the standard treatment options for patients with HGGs. With the continuous development and innovation of medical technology in recent years, the clinical treatment of HGGs has made some progress. Different groups of people should choose different dose fraction schemes, in order to realize the 
individualization of treatment schemes, and provide more benefits to patients. For older patients, preliminary data suggest that using a shorter course of radiotherapy may benefit. For HGG patients, it is feasible to integrate different imagings, such as PET and MRI, with precise radiotherapy planning. At present, the optimal radiotherapy dose, the fraction model, and how to achieve individualized radiotherapy remains unclear. Hence, further research is still needed. In view of the poor prognosis of this disease, patients should be encouraged to participate in properly conducted experimental studies.

\section{Acknowledgments}

We are particularly grateful to all the people who have given us help on our article.

Funding: None.

\section{Footnote}

Conflicts of Interest: Both authors have completed the ICMJE uniform disclosure form (available at http://dx.doi. org/10.21037/tcr-20-1891). The authors have no conflicts of interest to declare.

Ethical Statement: The authors are accountable for all aspects of the work in ensuring that questions related to the accuracy or integrity of any part of the work are appropriately investigated and resolved.

Open Access Statement: This is an Open Access article distributed in accordance with the Creative Commons Attribution-NonCommercial-NoDerivs 4.0 International License (CC BY-NC-ND 4.0), which permits the noncommercial replication and distribution of the article with the strict proviso that no changes or edits are made and the original work is properly cited (including links to both the formal publication through the relevant DOI and the license). See: https://creativecommons.org/licenses/by-nc-nd/4.0/.

\section{References}

1. Nabors LB, Portnow J, Ammirati M, et al. Central nervous system cancers, version 2.2014. Featured updates to the NCCN Guidelines. J Natl Compr Canc Netw 2014;12:1517-23.

2. Weller $M$, van den Bent $M$, Tonn JC, et al. European Association for Neuro-Oncology (EANO) guideline on the diagnosis and treatment of adult astrocytic and oligodendroglial gliomas. Lancet Oncol 2017;18:e315-29.

3. Omuro A, DeAngelis LM. Glioblastoma and other malignant gliomas: a clinical review. JAMA 2013;310:1842-50.

4. Ostrom QT, Gittleman H, Liao P, et al. CBTRUS statistical report: primary brain and central nervous system tumors diagnosed in the United States in 2007-2011. Neuro Oncol 2014;16:iv1-iv63.

5. Stupp R, Mason WP, van den Bent MJ, et al. Radiotherapy plus concomitant and adjuvant temozolomide for glioblastoma. N Engl J Med 2005;352:987-96.

6. Cairncross G, Wang M, Shaw E, et al. Phase III trial of chemoradiotherapy for anaplastic oligodendroglioma: long-term results of RTOG 9402. J Clin Oncol 2013;31:337-43.

7. Sun MZ, Oh T, Ivan ME, et al. Survival impact of time to initiation of chemoradiotherapy after resection of newly diagnosed glioblastoma. J Neurosurg 2015;122:1144-50.

8. Ten Haken RK, Thornton AF Jr, Sandler HM, et al. A quantitative assessment of the addition of MRI to CTbased, 3-D treatment planning of brain tumors. Radiother Oncol 1992;25:121-33.

9. Thibouw D, Truc G, Bertaut A, et al. Clinical and dosimetric study of radiotherapy for glioblastoma: threedimensional conformal radiotherapy versus intensitymodulated radiotherapy. J Neurooncol 2018;137:429-38.

10. Narayana A, Yamada J, Berry S, et al. Intensity-modulated radiotherapy in high-grade gliomas: clinical and dosimetric results. Int J Radiat Oncol Biol Phys 2006;64:892-7.

11. Toledo M, Sarria-Estrada S, Quintana M, et al. Prognostic implications of epilepsy in glioblastomas. Clin Neurol Neurosurg 2015;139:166-71.

12. Amelio D, Lorentini S, Schwarz M, et al. Intensitymodulated radiation therapy in newly diagnosed glioblastoma: a systematic review on clinical and technical issues. Radiother Oncol 2010;97:361-9.

13. Hermanto U, Frija EK, Lii MJ, et al. Intensity-modulated radiotherapy (IMRT) and conventional three-dimensional conformal radiotherapy for high-grade gliomas: does IMRT increase the integral dose to normal brain? Int J Radiat Oncol Biol Phys 2007;67:1135-44.

14. Shaffer R, Nichol AM, Vollans E, et al. A comparison of volumetric modulated arc therapy and conventional intensity-modulated radiotherapy for frontal and temporal high-grade gliomas. Int J Radiat Oncol Biol Phys 2010;76:1177-84.

15. Panet-Raymond V, Ansbacher W, Zavgorodni S, et al. Coplanar versus noncoplanar intensity-modulated 
radiation therapy (IMRT) and volumetric-modulated arc therapy (VMAT) treatment planning for fronto-temporal high-grade glioma. J Appl Clin Med Phys 2012;13:3826.

16. Chang JY, Zhang W, Komaki R, et al. Long-term outcome of phase I/II prospective study of dose-escalated proton therapy for early-stage non-small cell lung cancer. Radiother Oncol 2017;122:274-80.

17. Grewal AS, Schonewolf C, Min EJ, et al. Four-Year Outcomes From a Prospective Phase II Clinical Trial of Moderately Hypofractionated Proton Therapy for Localized Prostate Cancer. Int J Radiat Oncol Biol Phys 2019;105:713-22.

18. Robinson CG, Contreras J, Dewees TA, et al. Phase I Study of Accelerated Hypofractionated Proton Therapy and Chemotherapy for Locally Advanced Non-Small Cell Lung Cancer (LA-NSCLC). Int J Radiat Oncol Biol Phys 2018;102:S17.

19. Verma V, Rwigema JM, Malyapa RS, et al. Systematic assessment of clinical outcomes and toxicities of proton radiotherapy for reirradiation. Radiother Oncol 2017;125:21-30.

20. Adeberg S, Bernhardt D, Harrabi SB, et al. Sequential proton boost after standard chemoradiation for high-grade glioma. Radiother Oncol 2017;125:266-72.

21. Vora SA, Gondi V, Tseng YD, et al. Safety and Efficacy of Proton Beam Therapy for High-Grade Glioma: Initial Report From the Proton Collaborative Group (PCG). Int J Radiat Oncol Biol Phys 2018;102:E362.

22. Server A, Kulle B, Maehlen J, et al. Quantitative apparent diffusion coefficients in the characterization of brain tumors and associated peritumoral edema. Acta Radiol 2009;50:682-9.

23. Nakai T, Muraki S, Bagarinao E, et al. Application of independent component analysis to magnetic resonance imaging for enhancing the contrast of gray and white matter. Neuroimage 2004;21:251-60.

24. Gauvain KM, McKinstry RC, Mukherjee P, et al. Evaluating pediatric brain tumor cellularity with diffusiontensor imaging. AJR Am J Roentgenol 2001;177:449-54.

25. Park JY, Suh TS, Lee JW, et al. Dosimetric Effects of Magnetic Resonance Imaging-assisted Radiotherapy Planning: Dose Optimization for Target Volumes at High Risk and Analytic Radiobiological Dose Evaluation. J Korean Med Sci 2015;30:1522-30.

26. Papageorgiou TS, Chourmouzi D, Drevelengas A, et al. Diffusion Tensor Imaging in brain tumors: A study on gliomas and metastases. Phys Med 2015;31:767-73.

27. Jena R, Price SJ, Baker C, et al. Diffusion tensor imaging: possible implications for radiotherapy treatment planning of patients with high-grade glioma. Clin Oncol (R Coll Radiol) 2005;17:581-90.

28. Bian Y, Meng L, Peng J, et al. Effect of radiochemotherapy on the cognitive function and diffusion tensor and perfusion weighted imaging for high-grade gliomas: A prospective study. Sci Rep 2019;9:5967.

29. Kwee TC, Donswijk ML. Application of Advanced MR Imaging Techniques and the Evolving Role of PET/MR Imaging in Neuro-oncology. PET Clin 2013;8:183-99.

30. Cheng LL, Chang IW, Louis DN, et al. Correlation of high-resolution magic angle spinning proton magnetic resonance spectroscopy with histopathology of intact human brain tumor specimens. Cancer Res 1998;58:1825-32.

31. Elias AE, Carlos RC, Smith EA, et al. MR spectroscopy using normalized and non-normalized metabolite ratios for differentiating recurrent brain tumor from radiation injury. Acad Radiol 2011;18:1101-8.

32. Kostenikov NA, Tiutin LA, Fadeev NP, et al. Differential diagnosis of brain gliomas by positron emission tomography using various radiopharmaceuticals. Vestn Rentgenol Radiol 2014;(5):13-8.

33. Lohmann P, Herzog H, Rota Kops E, et al. Dual-timepoint O-(2-[(18)F]fluoroethyl)-L-tyrosine PET for grading of cerebral gliomas. Eur Radiol 2015;25:3017-24.

34. Rieken S, Habermehl D, Giesel FL, et al. Analysis of FETPET imaging for target volume definition in patients with gliomas treated with conformal radiotherapy. Radiother Oncol 2013;109:487-92.

35. Piroth MD, Holy R, Pinkawa M, et al. Prognostic impact of postoperative, pre-irradiation (18)F-fluoroethyl-1tyrosine uptake in glioblastoma patients treated with radiochemotherapy. Radiother Oncol 2011;99:218-24.

36. Munck Af Rosenschold P, Costa J, Engelholm SA, et al. Impact of [18F]-fluoro-ethyl-tyrosine PET imaging on target definition for radiation therapy of high-grade glioma. Neuro Oncol 2015;17:757-63.

37. Puttick S, Bell C, Dowson N, et al. PET, MRI, and simultaneous PET/MRI in the development of diagnostic and therapeutic strategies for glioma. Drug Discov Today 2015;20:306-17.

38. Grosu AL, Weber WA, Franz M, et al. Reirradiation of recurrent high-grade gliomas using amino acid PET (SPECT)/CT/MRI image fusion to determine gross tumor volume for stereotactic fractionated radiotherapy. Int J Radiat Oncol Biol Phys 2005;63:511-9.

39. Brandes AA, Tosoni A, Franceschi E, et al. Glioblastoma 
in adults. Crit Rev Oncol Hematol 2008;67:139-52.

40. Cabrera AR, Kirkpatrick JP, Fiveash JB, et al. Radiation therapy for glioblastoma: Executive summary of an American Society for Radiation Oncology EvidenceBased Clinical Practice Guideline. Pract Radiat Oncol 2016;6:217-25.

41. Minniti G, Amelio D, Amichetti M, et al. Patterns of failure and comparison of different target volume delineations in patients with glioblastoma treated with conformal radiotherapy plus concomitant and adjuvant temozolomide. Radiother Oncol 2010;97:377-81.

42. Tsien C, Moughan J, Michalski JM, et al. Phase I threedimensional conformal radiation dose escalation study in newly diagnosed glioblastoma: Radiation Therapy Oncology Group Trial 98-03. Int J Radiat Oncol Biol Phys 2009;73:699-708.

43. Souhami L, Seiferheld W, Brachman D, et al. Randomized comparison of stereotactic radiosurgery followed by conventional radiotherapy with carmustine to conventional radiotherapy with carmustine for patients with glioblastoma multiforme: report of Radiation Therapy Oncology Group 93-05 protocol. Int J Radiat Oncol Biol Phys 2004;60:853-60.

44. Kong DS, Lee JI, Park K, et al. Efficacy of stereotactic radiosurgery as a salvage treatment for recurrent malignant gliomas. Cancer 2008;112:2046-51.

45. Imber BS, Kanungo I, Braunstein S, et al. Indications and Efficacy of Gamma Knife Stereotactic Radiosurgery for Recurrent Glioblastoma: 2 Decades of Institutional Experience. Neurosurgery 2017;80:129-39.

46. Larson EW, Peterson HE, Lamoreaux WT, et al. Clinical outcomes following salvage Gamma Knife radiosurgery for recurrent glioblastoma. World J Clin Oncol 2014;5:142-8.

47. Morris SL, Zhu P, Rao M, et al. Gamma Knife Stereotactic Radiosurgery in Combination with Bevacizumab for Recurrent Glioblastoma. World Neurosurg 2019;127:e523-33.

48. Abbassy M, Missios S, Barnett GH, et al. Phase I Trial of Radiosurgery Dose Escalation Plus Bevacizumab in Patients with Recurrent/Progressive Glioblastoma. Neurosurgery 2018;83:385-92.

49. Corn BW, Curran WJ Jr, Shrieve DC, et al. Stereotactic radiosurgery and radiotherapy: new developments and new directions. Semin Oncol 1997;24:707-14.

50. Cardinale R, Won M, Choucair A, et al. A phase II trial of accelerated radiotherapy using weekly stereotactic conformal boost for supratentorial glioblastoma multiforme: RTOG 0023. Int J Radiat Oncol Biol Phys
2006;65:1422-8.

51. Fogh SE, Andrews DW, Glass J, et al. Hypofractionated stereotactic radiation therapy: an effective therapy for recurrent high-grade gliomas. J Clin Oncol 2010;28:3048-53.

52. Tsien C, Pugh S, Dicker AP, et al. Randomized Phase II Trial of Re-Irradiation and Concurrent Bevacizumab versus Bevacizumab Alone as Treatment for Recurrent Glioblastoma (NRG Oncology/RTOG 1205): Initial Outcomes and RT Plan Quality Report. Int J Radiat Oncol Biol Phys 2019;105:S78.

53. Malmström A, Grønberg BH, Marosi C, et al.

Temozolomide versus standard 6-week radiotherapy versus hypofractionated radiotherapy in patients older than 60 years with glioblastoma: the Nordic randomised, phase 3 trial. Lancet Oncol 2012;13:916-26.

54. Biau J, Chautard E, De Schlichting E, et al. Radiotherapy plus temozolomide in elderly patients with glioblastoma: a "real-life" report. Radiat Oncol 2017;12:197.

55. Roa W, Kepka L, Kumar N, et al. International Atomic Energy Agency Randomized Phase III Study of Radiation Therapy in Elderly and/or Frail Patients With Newly Diagnosed Glioblastoma Multiforme. J Clin Oncol 2015;33:4145-50.

56. Perry JR, Laperriere N, O'Callaghan CJ, et al. ShortCourse Radiation plus Temozolomide in Elderly Patients with Glioblastoma. N Engl J Med 2017;376:1027-37.

57. Thilmann C, Zabel A, Grosser KH, et al. Intensitymodulated radiotherapy with an integrated boost to the macroscopic tumor volume in the treatment of high-grade gliomas. Int J Cancer 2001;96:341-9.

58. Cho KH, Kim JY, Lee SH, et al. Simultaneous integrated boost intensity-modulated radiotherapy in patients with high-grade gliomas. Int J Radiat Oncol Biol Phys 2010;78:390-7.

59. Ge X, Xue X, Liu H, et al. Treatment of high-grade gliomas using escalating doses of hypofractionated simultaneous integrated boost-intensity-modulated radiation therapy in combination with temozolomide: A modified Phase I clinical trial. J Cancer Res Ther 2018;14:1482-91.

60. Mallick S, Kunhiparambath H, Gupta S, et al. Hypofractionated accelerated radiotherapy (HART) with concurrent and adjuvant temozolomide in newly diagnosed glioblastoma: a phase II randomized trial (HART-GBM trial). J Neurooncol 2018;140:75-82.

61. Laprie A, Ken S, Filleron T, et al. Dose-painting multicenter phase III trial in newly diagnosed glioblastoma: 
the SPECTRO-GLIO trial comparing arm A standard radiochemotherapy to arm $B$ radiochemotherapy with simultaneous integrated boost guided by MR spectroscopic imaging. BMC Cancer 2019;19:167.

62. Weller M, Pfister SM, Wick W, et al. Molecular neurooncology in clinical practice: a new horizon. Lancet Oncol 2013;14:e370-9.

63. Wick W, Wiestler B, Platten M. Treatment of anaplastic glioma. Cancer Treat Res 2015;163:89-101.

64. Hegi ME, Diserens AC, Gorlia T, et al. MGMT gene silencing and benefit from temozolomide in glioblastoma. N Engl J Med 2005;352:997-1003.

65. Eckel-Passow JE, Lachance DH, Molinaro AM, et al. Glioma Groups Based on 1p/19q, IDH, and TERT Promoter Mutations in Tumors. N Engl J Med 2015;372:2499-508.

66. Juratli TA, Lautenschläger T, Geiger KD, et al. Radiochemotherapy improves survival in IDH-mutant, $1 \mathrm{p} / 19 \mathrm{q}$ non-codeleted secondary high-grade astrocytoma patients. J Neurooncol 2015;124:197-205.

67. Houillier C, Wang X, Kaloshi G, et al. IDH1 or IDH2 mutations predict longer survival and response to temozolomide in low-grade gliomas. Neurology

Cite this article as: Zhang Y, Wang J. Research progress on radiotherapy technology and dose fraction scheme for advanced gliomas. Transl Cancer Res 2020;9(12):7642-7651. doi: 10.21037/ tcr-20-1891
2010;75:1560-6.

68. Leibel SA, Sheline GE. Radiation therapy for neoplasms of the brain. J Neurosurg 1987;66:1-22.

69. Douw L, Klein M, Fagel SS, et al. Cognitive and radiological effects of radiotherapy in patients with lowgrade glioma: long-term follow-up. Lancet Neurol 2009;8:810-8.

70. Tabrizi S, Yeap BY, Sherman JC, et al. Long-term outcomes and late adverse effects of a prospective study on proton radiotherapy for patients with low-grade glioma. Radiother Oncol 2019;137:95-101.

71. Gross JP, Powell S, Zelko F, et al. Improved neuropsychological outcomes following proton therapy relative to X-ray therapy for pediatric brain tumor patients. Neuro Oncol 2019;21:934.

72. Surma-aho O, Niemela M, Vilkki J, et al. Adverse longterm effects of brain radiotherapy in adult low-grade glioma patients. Neurology 2001;56:1285-90.

73. Richard NM, Bernstein LJ, Mason WP, et al. Cognitive rehabilitation for executive dysfunction in brain tumor patients: a pilot randomized controlled trial. J Neurooncol 2019;142:565. 